\title{
A NEW SIMPLIFIED APPROACH TO FIND THE EQUIVALENT RESISTANCE OF ANY COMPLEX EQUAL RESISTIVE NETWORK
}

\author{
Karan Aneja \\ Electrical Engineering Deptt. \\ Dehradun Institute of Technology \\ Dehradun, India
}

\author{
Vipin Kumar \\ Electronics \& communication Engineering Deptt. \\ Tula's Institute, Engineering \& Management \\ College, Dehradun, India
}

\begin{abstract}
As we are familiar with the star-delta transformation to determining the equivalent resistance [4] between any two terminals of a complex network and to find the overall current of a closed circuit, but sometimes circuit become too complex such that it consumes a lots of time while solving the network to find the equivalent resistance between two known terminals. So, here we have developed a new simplified approach for the solution to find the equivalent resistance between the desired terminals for equal resistance network and to find the overall current in a closed circuit.
\end{abstract}

Keywords: star-delta transformation, equivalent resistance, complex network, open circuit, (key words)

\section{INTRODUCTION}

Whenever we have a complex resistive networks/circuits such that we cannot determine which branches of resistance are in parallel or in series, for that networks we have star-delta transformation approach[4] to find the equivalent resistance of that resistive networks. Star-delta transformation simply derives the relations between star network to delta and delta to star network. By using these relations we can convert a complex network to simplest network as per the convenience in series-parallel combination and thus we can calculate the overall resistance of any network. It's quite worthy approach to simplify the circuit/network but as the circuit becomes more complex it consumes more time while solving the problem[2]. As we have no other approach to simplify the complex networks, here we have generated a new methodology to solve the complex equal resistance network. The main purpose of introducing this new approach is to simplify the circuit more efficiently and to minimize the time consumption while attempting to solve it.

\section{NEW METHODOLOGY}

We have generated a basic technique followed by number of instructions by following them we can have the solution for the complex network and can find the equivalent resistance of equal resistive network and the overall circuit current. We have shown our method approach by designing the number of complex circuits having equal resistance and comparing it with star -delta transformation approach. It is quite interesting to note that the result obtained by this methodology is exact replica of the one approached by /star-Delta Transformation Technique without any error.

\section{PROPOSED ALGORITHM}

There have three instructions in this proposed algorithm by executing them step by step we can find the equivalent resistance of a complex network and can find the circuit current. We can understand them by following an example.
* Keep intact all the branches connected to the terminals across which the equivalent resistance to be find out. Let these terminals be represented by " $\mathrm{A}$ " and' B".

* Search for that resistive branch in a given network such that it would be a null detection branch of the four- arm bridge.

* After that open that branch by removing the resistance between those terminals. Let these terminals be represented by "C" and " $\mathrm{D}$ ".

\subsection{Complex circuit example (1)}

Complex network/circuit shown in figure (1) to find resistance between terminals $\mathrm{A}$ and $\mathrm{B}$.

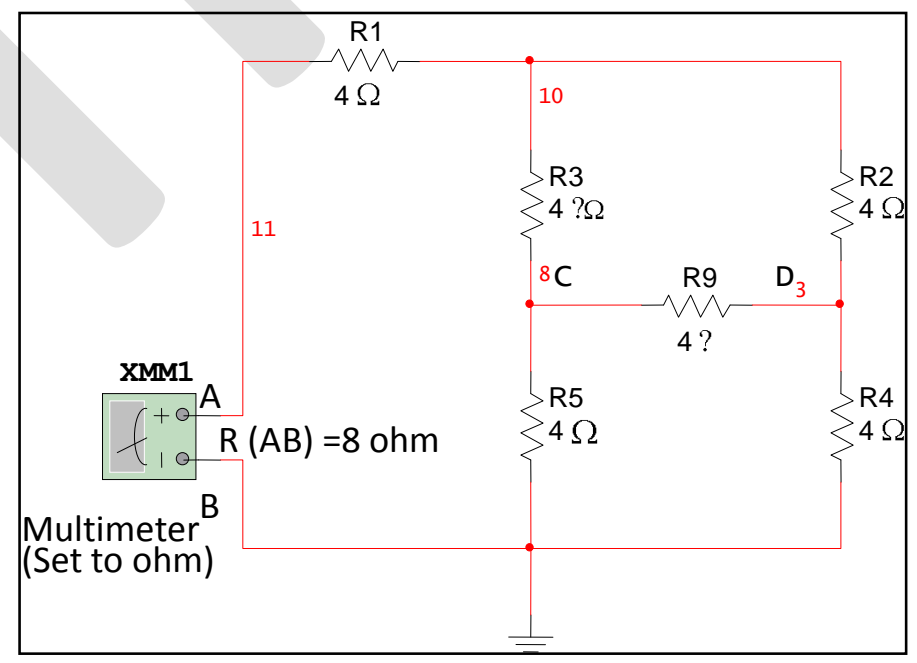

Figure (1)

By star-delta transformation approach[1], by using the star to delta and delta to star conversion relations as given below we can simplify the network as per the convenience and its equivalent resistance can be calculated and for the above network shown in figure (1) it comes out to be $8 \mathrm{ohm}$ between terminals A and B.

Figure (2) shows the star-delta transformation three terminal network and its equations for transformation is given below. 

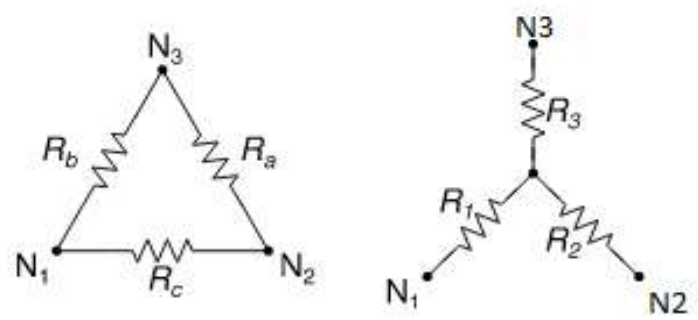

Figure (2)

Equations for transformation from delta to star network are

$\mathrm{R}_{1}=\frac{\mathrm{R}_{\mathrm{b}} \mathrm{R}_{\mathrm{c}}}{\mathrm{R}_{\mathrm{a}}+\mathrm{R}_{\mathrm{b}}+\mathrm{R}_{\mathrm{c}}} \quad, \quad \mathrm{R}_{2}=\frac{\mathrm{R}_{\mathrm{a}} \mathrm{R}_{\mathrm{c}}}{\mathrm{R}_{\mathrm{a}}+\mathrm{R}_{\mathrm{b}}+\mathrm{R}_{\mathrm{c}}}, \quad \mathrm{R}_{3}=\frac{\mathrm{R}_{\mathrm{a}} \mathrm{R}_{\mathrm{b}}}{\mathrm{R}_{\mathrm{a}}+\mathrm{R}_{\mathrm{b}}+}$ $\mathrm{R}_{\mathrm{c}}$

Equations for transformation from star to delta network are

$R_{a}=\frac{R_{1} R_{2}+R_{2} R_{3}+R_{3} R_{1}}{R_{1}}, \quad R_{b}=\frac{R_{1} R_{2}+R_{2} R_{3}+R_{3} R_{1}}{R_{2}}$

$\mathrm{R}_{\mathrm{c}}=\mathrm{R}_{1} \mathrm{R}_{2}+\mathrm{R}_{2} \mathrm{R}_{3}+\mathrm{R}_{3} \mathrm{R}_{1}$

$$
\mathrm{R}_{3}
$$

Now, by approaching the methodology described above, if we follow those instructions we can find that the equivalent resistance of the network in figure (1) between terminals A and B will be the same as that of the result out from the star delta transformation approach i.e. $8 \mathrm{ohm}$.

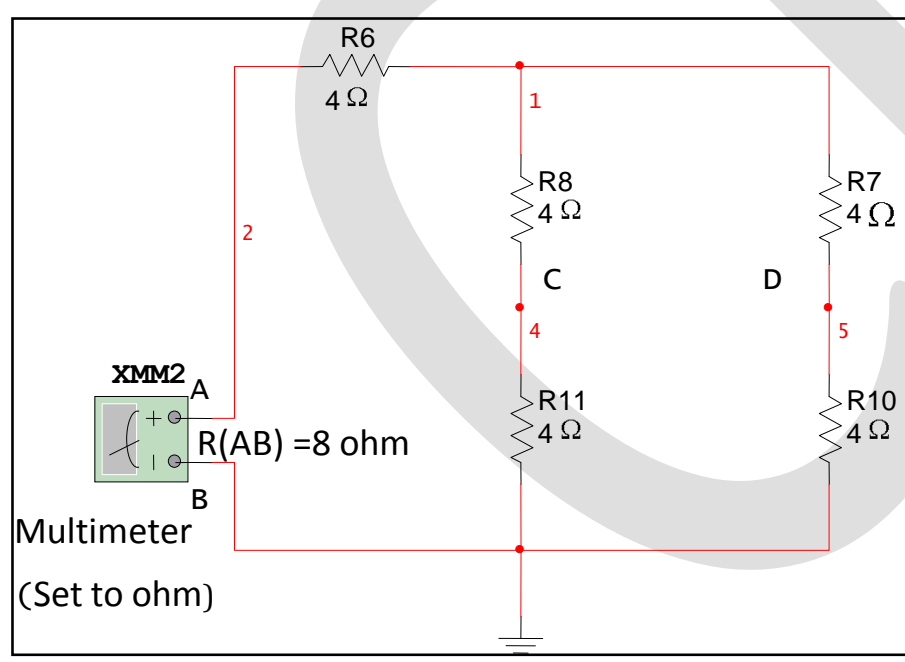

Figure (3)

It is observed in figure (3) that the branch between terminals C \& D is opened as it is null detection branch of the four-arm bridge network.

NOTE: whatever be the value of the null detection branch resistance the overall network resistance will remain always be same.

Some other complex networks are shown here and both techniques are applied to them and it will be observed that the network equivalent resistance will remain the same.

\subsection{Complex circuit example (2)}

A complex network shown in figure (4) [4], by star-delta transformation approach the equivalent resistance between terminal $\mathrm{A}$ and $\mathrm{B}$ comes out to be $0.5 \mathrm{ohm}$.

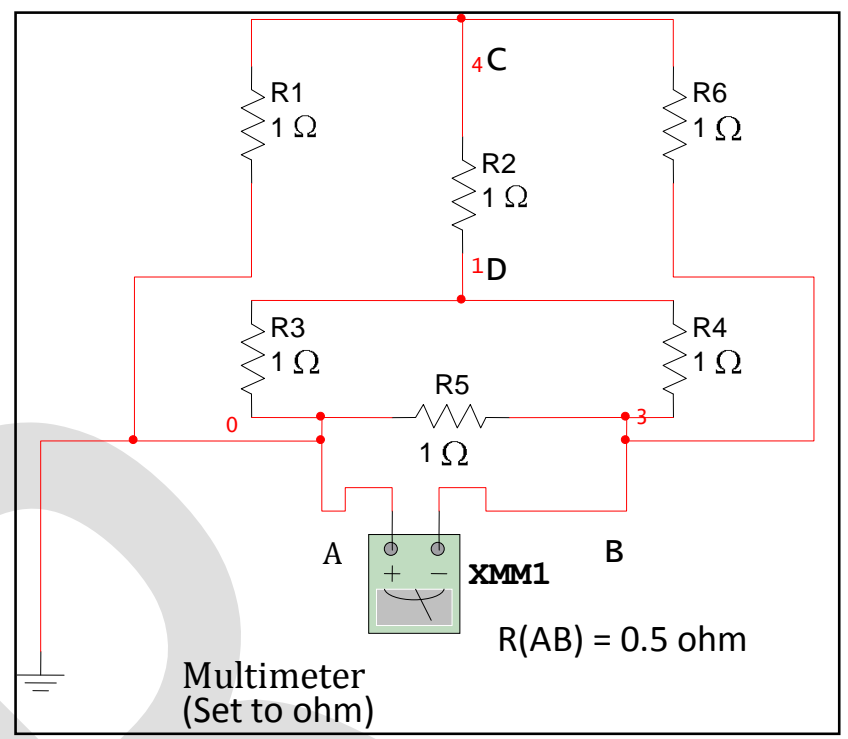

Figure (4)

Now, by approaching the algorithm proposed, we find that equivalent resistance remains the same $0.5 \mathrm{ohm}$ as shown in figure (5).

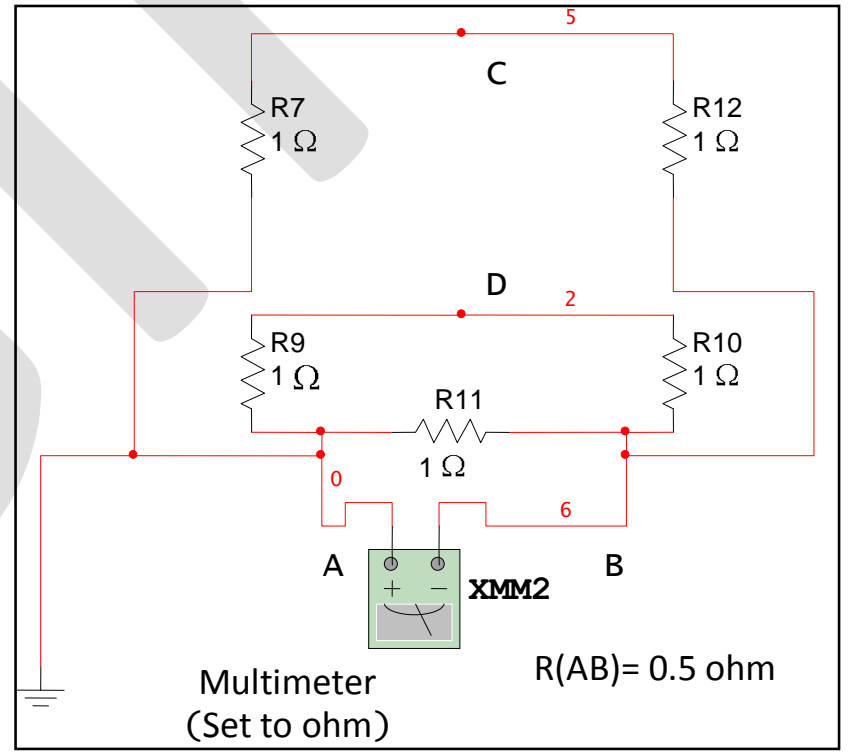

Figure (5)

\subsection{Complex circuit Analysis example(3)}

Let's take more complex network as shown in figure (6) on next page. The same procedure is applied to this network and matched with the star-delta approach. By following the proposed algorithm the given network is sorted out and compared its result with the predefined approach. 


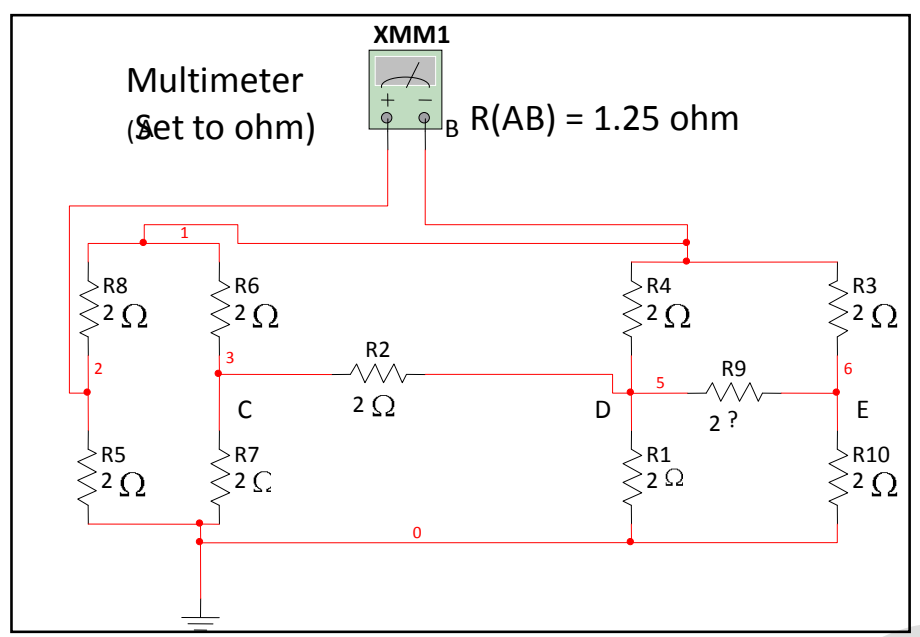

Figure (6)

By star-delta approach the equivalent resistance between terminals $\mathrm{A}$ and $\mathrm{B}$ comes out to be $1.25 \mathrm{ohm}$.

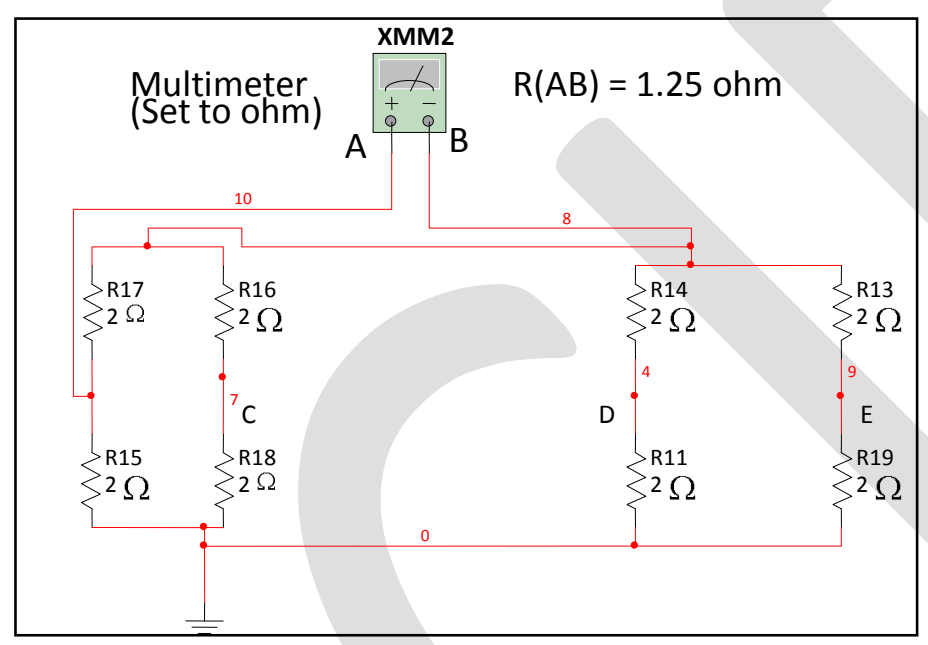

Figure (7)

Figure (7) shows the approach of the new methodology defined and it is found that the results are the same for both the cases.

\section{RESULT AND CONCLUSION}

Thus, it is observed that any complex network having equal resistance can be simplified by employing the methodology described in this paper. We can find the equivalent resistance between the desired terminals and also we can determine the closed circuit current by placing a voltage source across those terminals where we have to find the equivalent resistance. What we concluded by employing this methodology is that the time efficiency i.e. this technique does not consumes too much time to solve the complex network as it is observed in star delta transformation approach. In star-delta transformation approach, firstly we have to search for the star or delta connected network and then convert them in a convenient way by using the star-delta transformation equations and simplify the network step by step redesigning it till the complete solution is not obtained but in this proposed methodology, there is no need of cramming any relations and it needs hardly one or two steps to simplify the network.

\section{FUTURE SCOPE}

The methodology employed in this paper is limited to the equal resistive network but further work can be done for the unequal resistive network and also for the finding the equivalent impendence of A.C. network. This technique would be quite worthy if it is getting successful for both the D.C as well as A.C network.

\section{REFERENCES}

[1] A.E. Kennelly, Equivalence of triangles and stars in conducting networks, Electrical World and Engineer, vol. 34, pp. 413-414, 1899.I..

[2] Grosh, Doris Lloyd, "Comments on the Delta-Star Problem", IEEE Transactions on Reliability, Volume: R-32 ,Issue: 4Page(s): 391- 394,oct-1983.

[3] Gadani, J.P. press., "System Effectiveness Evaluation Using Star and Delta Transformations", IEEE Transactions on Reliability, Volume:R-30 , Issue: 1, Page(s): $43 \quad$ - $\quad 47$, 1981.

[4] Robert L. Boylestad "Introductory Circuit Analysis (10th Edition)" ISBN: 013097417X. 